\title{
Lower Extremity Muscle Activity during Different Types and Speeds of Underwater Movement
}

\author{
Koichi Kaneda, Hitoshi Wakabayashi, Daisuke Sato and Takeo Nomura \\ Institute of Comprehensive Human Sciences, University of Tsukuba
}

\begin{abstract}
To compare the activity of lower extremity muscles during land walking (LW), water walking (WW), and deepwater running (DWR), 9 healthy young subjects were tested at self-selected low, moderate, and high intensities for $8 \mathrm{sec}$ with two repetitions. Surface EMG electrodes were placed on the tibialis anterior (TA), soleus (SOL), medial gastrocnemius (GAS), rectus femoris (RF), and biceps femoris (BF). During DWR, the SOL and GAS activities were lower than LW and WW. The BF activities were higher during DWR than LW and WW. It was considered that the lower activity of SOL and GAS depended on water depth, and higher activity of BF occurred by greater flexion of the knee joint or extension of the hip joint during exercise. J Physiol Anthropol 26(2): 197-200, 2007 http://www.jstage.jst.go.jp/browse/jpa2

[DOI: 10.2114/jpa2.26.197]
\end{abstract}

Keywords: surface EMG, water walking, deep-water running, self-selected intensity, muscle activity

\section{Introduction}

Various water exercises exist for rehabilitation or fitness maintenance. In water, buoyancy acts against the body to reduce the load at the joints, while water viscosity requires the subject to exert greater force than when moving on land (Miyoshi et al., 2004).

An upright-floating situation in a water environment (feet separated from the swimming pool floor) is hard to experience in any other exercise environment. The typical form of uprightfloating exercise in water is deep-water running (DWR). The advantages of this exercise are that it can reduce the impact stress for lower limb joints and maintain aerobic fitness (Reilly et al., 2003). Studies have investigated motion analysis (Moening et al., 1993) and aerobic fitness (Svedenhag and Seger, 1992) during DWR and described its characteristics. Recently, DWR has been employed not only by athletes but also by elderly persons (Broman et al., 2006) for the purpose of aerobic fitness and recuperation from minor arthopedic injury.

However, there are few reports which investigate lower extremity muscle activity during DWR. Many previous studies have reported markedly higher cardiorespiratory responses or muscle activity during water walking than for land walking at the same speed (Migita et al., 1994; Hall et al., 1998; Kato et al., 2002). Green et al. (1990) conducted deep-water and treadmill walking with self-determined "slow," "moderate," and "quite fast" speeds, and concluded that the intensity of work should be assessed for each individual and exercise environment. As mentioned above, it was difficult to fix the physical and physiological intensity during exercise. Some studies have conducted water and land walking based on ratings of perceived exertion (RPE) or self-determined intensity (Fujishima and Shimizu, 2003; Miyoshi et al., 2004). From the perspective of group water exercise, it is very hard to control the exercise intensity accurately for each individual. Such exercise is therefore often conducted with selfdetermined intensity. To investigate the effect of water exercise on self-determined intensity is thus more appropriate. The purpose of this study was to compare lower extremity muscle activity during land walking (LW), water walking (WW), and deep-water running (DWR) at self-determined intensity.

\section{Methods}

Nine healthy young males participated in this experiment as subjects. Their mean age, height, weight, and \%fat were $24.9 \pm 2.2 \mathrm{yrs}, 172.0 \pm 3.8 \mathrm{~cm}, 69.3 \pm 3.7 \mathrm{~kg}$, and $19.4 \pm 4.1 \%$, respectively. Each subject's characteristics are presented in Table 1. Informed consent was obtained for this experiment. Subjects practiced to familiarize themselves with LW, WW, and DWR before the experiment. The subjects performed LW, WW, and DWR at self-determined low, moderate, and high intensities for $8 \mathrm{sec}$ with two repetitions. An aqua jogger (Aqua Jogger; Excel Sports Science Inc., Japan) was attached to the subject's waist during DWR, which enables subjects to seperate their feet from the bottom of the swimming pool.

The left lower extremity muscle activity of the tibialis anterior (TA), soleus (SOL), medial gastrocnemius (GAS), rectus femoris (RF), vastus lateralis (VL), and biceps femoris (BF) were measured during trials using surface electromyography (EMG). The skin cuticle was removed 
Table 1 Characteristics of each subject

\begin{tabular}{c|c:c:c:c}
\hline Subject & Age (yrs) & Height (cm) & Weight (kg) & \% Fat (\%) \\
\hline A & 27 & 174.2 & 67.4 & 16.5 \\
B & 21 & 168.5 & 72.0 & 16.2 \\
C & 27 & 177.1 & 67.6 & 18.2 \\
D & 25 & 173.6 & 64.1 & 15.5 \\
E & 25 & 171.7 & 66.5 & 20.0 \\
F & 26 & 172.3 & 75.4 & 27.6 \\
G & 21 & 175.0 & 73.3 & 16.3 \\
H & 25 & 163.4 & 72.2 & 25.6 \\
I & 27 & 172.0 & 65.6 & 18.6 \\
\hline AVE. & 24.9 & 172.0 & 69.3 & 19.4 \\
SD & 2.2 & 3.8 & 3.7 & 4.1 \\
\hline
\end{tabular}

carefully using a blood lancet (Blood Lancet; Asahi Polyslider Co. Ltd., Japan) and cleaned with alcohol wipes so that the inter-electrode impedance was less than $20 \mathrm{k} \Omega$. A pair of surface EMG electrodes ( $5 \mathrm{~mm}$ diameter) was placed on the muscle belly with an inter-electrode distance of $2.5 \mathrm{~cm}$. The electrodes were covered with transparent film (Dressing tape No. 100; As One Corp., Japan) and putty for waterproofing. The EMG signals were telemetered via a multi-channel telemetry system (WEB-5500 Nihon Kohden multi-telemeter system; Nihon Kohden Corp., Japan) using a time constant of $0.03 \mathrm{~s}, 500 \mathrm{~Hz}$ high cut filter, $2 \mathrm{kHz}$ sampling rate.

The trials were videotaped with synchronization to the EMG. A digital video camera was placed on the left side of the subject so that it allowed coverage of one cycle at a $30 \mathrm{~Hz}$ frame rate. Data were collected from one cycle of the videotaped picture, from heel contact to the next heel contact in LW and WW, and from the maximum knee drive (as a maximal flexion of the hip joint) to the next maximum knee drive in DWR. The Root Mean Square (RMS) of one cycle was calculated using the following procedure: The EMG signals of one cycle were squared and averaged, then the square root of the averaged value was taken. Since the time required to move one cycle was different with each trial, to calculate the RMS was regarded as the most appropriate analysis. Calculated all muscle RMS values were presented as percentages of maximum voluntary contraction (\%MVC) in each muscle. Before the locomotion trials, maximal voluntary contraction (MVC) in each muscle was performed on land according to the muscle testing instructions of the Anatomical Guide (Perotto et al., 1994). The duration of this MVC isometric contraction test was set at $5 \mathrm{sec}$ for each muscle. The water temperature was set at $27^{\circ} \mathrm{C}$ and the water depth was set at $1.1 \mathrm{~m}$ throughout the experiment.

A one-way repeated measures analysis of variance (ANOVA) was used to compare the LW, WW, and DWR at each intensity. If the ANOVA was significant, Tukey's test was used as a post hoc test. Statistical significance was inferred at $p<0.05$.

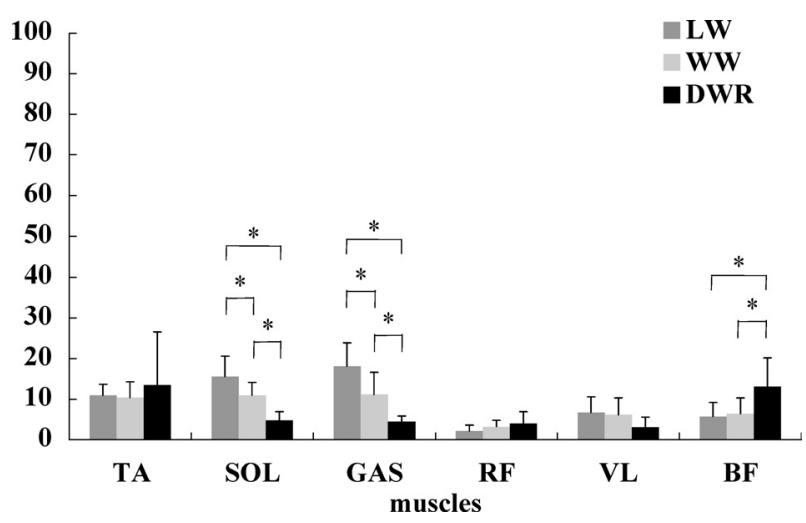

Fig. $1 \%$ MVC value during one cycle at low intensity of six muscles TA, tibialis anterior; SOL, soleus; GAS, medial gastrocnemius; RF, rectus femoris; VL, vastus lateralis; $\mathrm{BF}$, biceps femoris. *Significant difference between two conditions $(p<0.05)$.

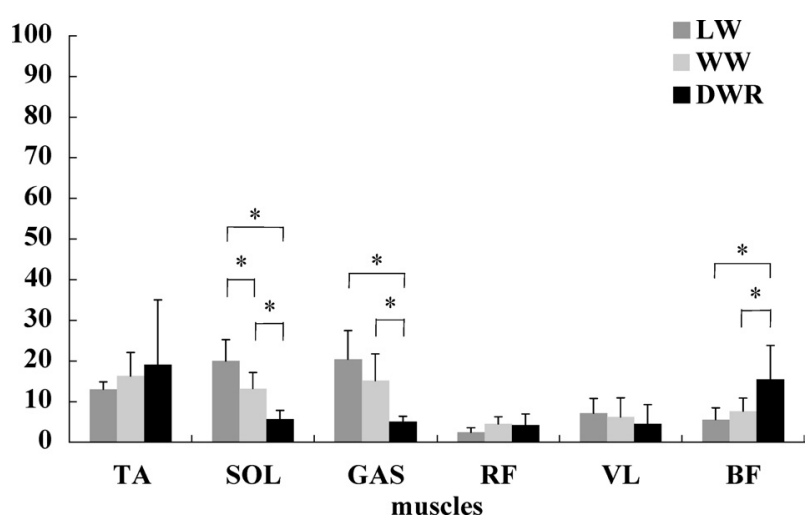

Fig. $2 \%$ MVC value during one cycle at moderate intensity of six muscles

TA, tibialis anterior; SOL, soleus; GAS, medial gastrocnemius; RF, rectus femoris; VL, vastus lateralis; $\mathrm{BF}$, biceps femoris.

*Significant difference between two conditions $(p<0.05)$.

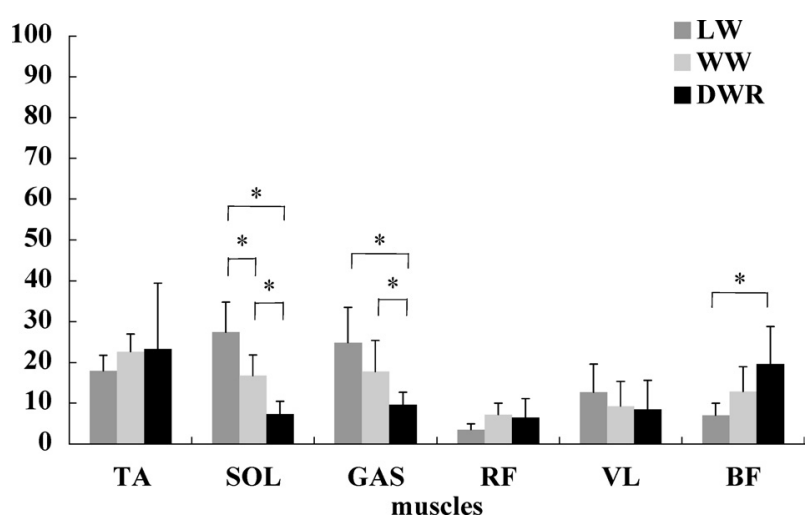

Fig. $3 \% \mathrm{MVC}$ value during one cycle at high intensity of six muscles TA, tibialis anterior; SOL, soleus; GAS, medial gastrocnemius; RF, rectus femoris; VL, vastus lateralis; BF, biceps femoris.

*Significant difference between two conditions $(p<0.05)$.

\section{Results}

Figure 1 shows the group mean of \%MVC during one cycle 
at low intensity for the six muscles. Figure 2 and Figure 3 show the group mean of \%MVC at moderate and high intensity for six muscles. SOL and GAS showed significantly lower activity $(p<0.05)$ during DWR than LW and WW at all intensities. During WW, SOL showed significantly lower activity $(p<0.05)$ than LW at all intensities, and GAS showed significantly lower activity $(p<0.05)$ than LW at low intensity. BF showed significantly higher activity $(p<0.05)$ during DWR than LW and WW at low and moderate intensities, and than LW at high intensity $(p<0.05)$.

\section{Discussion}

The significantly lower activity of SOL and GAS during DWR than LW and WW at all intensities was reasonable because DWR uses no force that involves friction with the pool bottom. Kato et al. (2002) reported similar \%MVC between LW and WW of SOL and GAS at the speed of 0.4 and $0.6 \mathrm{~m} / \mathrm{s}$. However, they detected significantly higher \%MVC of SOL and GAS during WW than LW at $0.8 \mathrm{~m} / \mathrm{s}$. Masumoto et al. (2004) set the same level of energy expenditure between land treadmill and water treadmill with water current walking (water+cur). They reported not significant but slightly lower $\%$ MVC during water+cur than land treadmill of GAS. In the present study, the subjects walked with self-selected intensity, so the walking speed was probably slower during WW than LW because of the water resistance. Therefore, the present results of SOL and GAS showed lower \%MVC during WW than LW at all intensities; in particular, significantly lower $\% \mathrm{MVC}$ was seen at all intensities in SOL and at low intensity in GAS.

Moening et al. (1993) suggested that the joint angle of hip maximum flexion in the knee drive was greater during DWR than treadmill running. At the knee joint, the range of motion from the back swing to the knee drive was greater during DWR than treadmill running. Hip and knee joint flexion and range of motion were greater during DWR than treadmill running. Miyoshi et al. (2004) reported that the range of motion at the hip joint during WW was similar to land walking at a comfortable speed, and that the range of motion at the knee joint during WW was smaller than land walking. Nilsson et al. (1985) compared treadmill walking at speeds from $0.4 \mathrm{~m} / \mathrm{s}$ to $3.0 \mathrm{~m} / \mathrm{s}$ and treadmill running from $1.0 \mathrm{~m} / \mathrm{s}$ to $9.0 \mathrm{~m} / \mathrm{s}$. They reported that the range of motion change of the hip joint from the lowest to the highest speed was about four times larger in treadmill running than treadmill walking. But they indicated that the range of motion of the hip joint was somewhat larger in treadmill walking compared to treadmill running at the same speeds. They also reported significantly larger flexion of the knee joint during treadmill running than walking.

In this study, the \%MVC of BF was significantly higher during DWR than LW at all intensities and than WW at low and normal intensities; moreover, it was not significantly but slightly higher during DWR than WW at high intensity. The higher \%MVC of BF during DWR than LW and WW was attributable to the greater range of motion at the knee and hip joint during DWR. A motion analysis research directory comparing LW, WW, and DWR is required to elucidate this aspect more precisely.

DWR has come to prominence for aquatic exercise programs in recent days. From the results of the present study, DWR may have a positive effect for improving balance in elderly people. The elderly use a hip strategy more often than young adults when controlling balance (Woollacott, 1993), and activate hamstrings more than the young when attempting to enhance hip joint stabilization (Benjuya et al., 2004; Laughton et al., 2003). Some form of intervention research is needed to clarify the effects on the ability to balance in the elderly.

\section{References}

Benjuya N, Melzer I, Kaplanski J (2004) Aging-induced shifts from a reliance on sensory input to muscle cocontraction during balanced standing. J Gerontol Med Sci 59A: 166-171

Broman G, Quintana M, Engardt M, Gullstrand L, Jansson E, Kaijser L (2006) Older women's cardiovascular responses to deep-water running. J Aging and Physical Activity 14: $29-40$

Fujishima K, Shimizu T (2003) Body temperature, oxygen uptake and heart rate during walking in water and on land at an exercise intensity based on RPE in elderly men. J Physiol Anthropol 22: 83-88

Green J, Cable N, Elms N (1990) Heart rate and oxygen consumption during walking on land and in deep water. J Sports Med Phys Fitness 30: 49-52

Hall J, Macdonald I, Maddison P, O’Hare J (1998) Cardiorespiratory responses to underwater treadmill walking in healthy females. Eur J Appl Physiol Occup Physiol 77: 278-284

Kato T, Sugagima Y, Koeda M, Fukuzawa S, Kitagawa K (2002) Electromyogram activity of leg muscles during different types of underwater walking. Adv Exerc Sports Physiol 8: 39-44

Laughton C, Slavin M, Katdare K, Nolan L, Bean J, Kerrigan Dm Phillips E, Lipsitz L, Collins J (2003) Aging, muscle activity, and balance control: physiologic changes associated with balance impairment. Gait and Posture 18: 101-108

Masumoto K, Takasugi S, Hotta N, Fujishima K, Iwamoto Y (2004) Electromyographic analysis of walking in water in healthy humans. J Physiol Anthrop Appl Human Sci 23: 119-127

Migita T, Muraoka Y, Hotta N, Ogaki T, Kanaya S, Fujushima K, Masuda T (1994) Cardiorespiratory responses during water and land walking. Kurume $\mathrm{J}$ of Health \& Physical Education 2: 25-30 [In Japanese]

Miyoshi T, Shirota T, Yamamoto S, Nakazawa K, Akai M (2004) Effect of the walking speed to the lower limb joint angular displacements, joint moments and ground reaction forces during walking in water. Disability and Rehabilitation 
26: 724-732

Moening D, Shepardson L, Davis G (1993) Biomechanical comparison of water running and treadmill running. Isokinetics and Exercise Science 3: 207-215

Nilsson J, Thorstensson A, Halbertsma J (1985) Changes in leg movements and muscle activity with speed of locomotion and mode of progression in humans. Acta Physiol Scand 123: 457-475

Perotto A, Morrison D, Delagi E, Iazzetti J (1994) Anatomical guide for the electromyographer: The limbs and trunk, 3rd ed. New York, Charles C Thomas

Reilly T, Dowzer C, Cable N (2003) The physiology of deepwater running. J Sports Sci 21: 959-972

Svedenhag J, Seger J (1992) Running on land and in water: comparative exercise physiology. Med Sci Sports Exerc 24:
1155-1160

Woollacott M (1993) Age-related changes in posture and movement. J Gerontol 48: 56-60

This article was presented at the 8th International Congress of Physiological Anthropology, 2006 (ICPA 2006), in Kamakura, Japan

Received: September 29, 2006

Accepted: December 11, 2006

Correspondence to: Koichi Kaneda, Institute of Health and Sport Sciences, University of Tsukuba, 1-1-1, Tennodai, Tsukuba, Ibaraki, 305-8574, Japan

Phone: +81-29-853-6338

Fax: +81-29-853-6338

e-mail: s0530485@ipe.tsukuba.ac.jp 